\title{
Efectividad del sildenafilo en Atención Primaria
}

\author{
E. Cerrada Cerrada, I. O'Connor Basalo, F. García de Blas González*, \\ A. J. CABAllero Gallego, I. DEL CuRA GonzÁleZ, \\ M. A. BARAJAS GutiÉRREZ, G. G ARCíA SÁNCHEZ \\ Especialista en Medicina de Familia. *Doctora en Medicina y Especialista
}

en

Medicina de Familia. Centro de Salud Dr. Mendiguchía. Leganés. Madrid

\section{RESUMEN}

Fundamentos: no hay estudios que evalúen la utilidad del sildenafilo (Viagra®) en Atención Primaria.

Objetivos: valorar la efectividad del sildenafilo en hombres con disfunción eréctil $(D E)$ mediante un cuestionario y una pregunta de eficacia global, así como el grado de satisfacción de su pareja.

Diseño: estudio de intervención antes-después no aleatorio.

Emplazamiento: centro de salud urbano.

Pacientes: varones $\check{Z} 18$ años con DE Ž 6 meses de evolución.

Métodos: se recogieron variables sociodemográficas, las patologías asociadas, el grado de DE-mediante la administración pre y postratamiento del campo función eréctil del Índice Internacional de Función Eréctil (IIEF) más una pregunta de eficacia global postratamiento- y una pregunta pre y postratamiento del grado de satisfacción de la pareja. Se utilizó el test de Wilcoxon para valorar la mejoría en la puntuación del cuestionario.

Resultados: 45 varones aceptaron inicialmente participar en el estudio con una edad media de 56,8 (IC 95\%: 32,6-81) años, 11 con DE leve, 21 moderada y 13 grave. Doce sujetos rechazaron posteriormente realizar el tratamiento; 5 de ellos por miedo a secundarismos. Los 33 sujetos que lo completaron presentaron un IIEF inicial con valor medio de 12,73 puntos (IC 95\%: 11,22-14,24) y el IIEF final de 24 (IC 95\%: 21,47-26,53), siendo esta mejoría estadísticamente significativa $(p<0,0001)$ y 28 mejoraron subjetivamente. Tres pacientes presentaron cefalea y dos rubefacción facial. De las 23 parejas (mujeres) que participaron, 20 refirieron
Effectiveness of sildenafil in Primary Health Care

\section{ABSTRACT}

Backgrounds: there aren't studies about efectiveness of sildenafil in Primary Health Care.

Objectives: to evaluate the effectiveness of sildenafil citrate (Viagra ${ }^{T M}$ ) in men with erectile dys function (ED) through a questionnaire and a global efficacy question, and their couples' satisfaction degree.

Design: non-aleatory before-after interventional study.

Location: urban primary care center.

Patients: men Ž 18 years with ED of 6 months or longer.

Methods: socio-demographical variables, associated diseases, ED degree -through the administration before and after treatment of the erectile function field of the International Index of Erectile Function (IIEF) and an after treatment global efficacy question-and a before and after treatment question about their couples' satisfaction degree, were measured. The Wilcoxon test was used to evaluate the improvement at the questionnaire score.

Results: initially, 45 men accepted to participate in the study, with a mean age of 56,8 years ( $C I$ 95\%: 32,6-81); 11 with mild ED, 21 moderate and 13 severe. Twelve rejected to follow the treatment; five of them were afraid of adverse effects. The 33 subjects that finished the study presented an initial IIEF mean value of 12,73 (CI 95\%: 11,22-14,24) and at the end an IIEF score of 24 (CI 95\%: 21,4726,53). This improvement was statistically significant ( $p<0,0001)$. Twenty-eight experienced subjective improvement. Three patients suffered headache and 2 flush. Twenty of the 23 mates (all women) that participated in the study referred increase in their sexual satisfaction degree. 
aumento del grado de satisfacción.

Conclusiones: el sildenafilo ha demostrado ser efectivo en la población de estudio en concordancia con otros trabajos a pesar de las limitaciones del diseño y del tamaño muestral.

Palabras clave: Sildenafilo. Disfunción eréctil.
Conclusions: sildenafil has demonstrated to be effective in our study population, as it was in other studies though the limitations of the design and size of the study.

Key words: Sildenafil. Sexual dysfunctions. Impotence. Primary Health Care.

\section{INTRODUCCIÓN}

La disfunción eréctil (DE) se define como la incapacidad para alcanzar y mantener una erección con la rigidez suficiente para completar una relación sexual satisfactoria ${ }^{1}$. Tiene una prevalencia global estimada en nuestro país del $19 \%$ entre los varones de 25 a 70 años (DE leve 16,3\%, moderada 2,1 y grave $0,6 \%$ ). Es un trastorno relacionado con la edad, afectando a un $9 \%$ de los varones entre 25 y 39 años y hasta un $49 \%$ entre 60 y 70 años ${ }^{2}$. El origen de esta patología es multifactorial. En un $60 \%$ existe una causa orgánica, en un $30 \%$ un problema psicógeno, un $2 \%$ se asocia a fármacos y hasta en un tercio de los casos es mixta ${ }^{3}$. Su prevalencia es mayor en pacientes con enfermedades vasculares, neurológicas y urológicas, en tratamiento farmacológico (antidepresivos, antipsicóticos, simpaticolíticos, betabloqueantes, diuréticos y tratamientos hormonales) y en relación con el consumo de tóxicos como el tabaco, el alcohol y otras drogas.

El diagnóstico de la DE es fundamentalmente clínico, siendo importante valorar los factores psicológicos y la relación de pareja que pudieran estar interfiriendo en la función eréctil ${ }^{4}$. Su tratamiento debe basarse en el control de las enfermedades asociadas, la modificación de los hábitos tóxicos (tabaco, alcohol) y cuando sea posible la suspensión, disminución de la dosis o modificación de los fármacos que puedan facilitar su desarrollo. Durante años se han ensayado numerosas intervenciones terapéuticas, como dispositivos de vacío, medicación oral (trazodona, yohimbina), inyecciones intracavernosas de sustancias vasoactivas (PGE1, papaverina, fentolamina $)^{2,5,6}$ y diferentes técnicas quirúrgicas (corrección de desviaciones peneanas, implantación de prótesis para DE irreversibles) ${ }^{2}$ con resultados de eficacia, tolerancia y grado de cumplimentación variables 7 . En la última década, se ha utilizado el alprostadil por vía intracavernosa, mostrándose eficaz pero con mala adherencia al tratamiento (alto porcentaje de efectos secundarios, como dolor peneano, priapismo, fibrosis y hematomas, así como altas tasas de abandono de hasta un $41 \%)^{2,6}$, si bien recientemente se ha comercializado en gel intrauretral con resultados iniciales esperan- zadores $^{8}$. La aparición del sildenafilo oral ha levantado grandes expectativas de mejora del tratamiento farmacológico de esta patología y ha generado una demanda creciente por parte de los pacientes. $\mathrm{Su}$ eficacia ya ha sido ampliamente demostrada en diferentes ensayos clínicos ${ }^{9-12}$, habiéndose establecido unas contraindicaciones precisas para su empleo (pacientes en tratamiento con nitratos o con otros fármacos para tratar la $\mathrm{DE}$, pacientes con tensión arterial < 90/50 y en aquéllos a los que se les desaconseje la actividad sexual, como los enfermos con cardiopatías avanzadas) ${ }^{13}$. En nuestro país hasta la fecha sólo se han realizado estudios en atención especializada ${ }^{14}$, pero ninguno en Atención Primaria que permita conocer su efectividad en este medio. El objetivo de este estudio es valorar la efectividad del sildenafilo en varones que consultan por DE en un centro de salud, mediante un cuestionario y una pregunta de eficacia global, así como el incremento en el grado de satisfacción de su pareja tras la utilización del mismo.

\section{SUJETOS Y MÉTODOS}

Se diseñó un estudio de intervención no aleatorio antes-después, en un centro de salud urbano de Leganés (Madrid). Se incluyeron por muestreo consecutivo no probabilístico los varones Ž 18 años que consultaron por DE de $\check{Z} 6$ meses de evolución, procedentes de 8 consultas de medicina de familia. El periodo de estudio fue de 11 meses, desde mayo de 1999 hasta abril del 2000. Los pacientes respondieron al campo función eréctil del cuestionario Índice Internacional de Función Eréctil $\left(\right.$ IIEF) ${ }^{2}$ (Anexo 1), traducido a distintos idiomas ${ }^{15} \mathrm{y}$ que consta de 6 cuestiones, cada una con 5 respuestas puntuadas de 1 a 5 , indicando el 5 la mejor función eréctil. Este cuestionario permite confirmar el diagnóstico de DE (puntuación £25) y la clasifica en grado leve (17-25 puntos), moderado (11-16) o grave $(<10)$. Fueron excluidos los pacientes con antecedentes de enfermedad cardiovascular en los últimos 6 meses (cardiopatía isquémica, enfermedad cerebrovascular, tensión arterial $<90 / 50$ ), insuficiencia hepática o renal grave, tratamiento con nitratos, síndrome de dependencia alcohólica o 
Anexo 1

CAMPO FUNCIÓN ERÉCTIL DEL CUESTIONARIO ÍNDICE INTERNACIONAL DE FUNCIÓN ERÉCTIL (IIEF)

\begin{tabular}{|c|c|c|c|c|c|c|}
\hline & $\begin{array}{l}\text { Sin } \\
\text { actividad } \\
\text { sexual }\end{array}$ & $\begin{array}{l}\text { Casi } \\
\text { nunca } 0 \\
\text { nunca }\end{array}$ & $\begin{array}{l}\text { Menos de } \\
\text { la mitad } \\
\text { de las } \\
\text { veces }\end{array}$ & $\begin{array}{l}\text { La mitad } \\
\text { de las } \\
\text { veces }\end{array}$ & $\begin{array}{l}\text { Más de } \\
\text { la mitad } \\
\text { de las } \\
\text { veces }\end{array}$ & $\begin{array}{l}\text { Casi } \\
\text { siempre } 0 \\
\text { siempre }\end{array}$ \\
\hline $\begin{array}{l}\text { 1. Durante los últimos } 6 \text { meses, } \\
\text { icon qué frecuencia logró una erección } \\
\text { durante la actividad sexual? }\end{array}$ & 0 & 1 & 2 & 3 & 4 & 5 \\
\hline $\begin{array}{l}\text { 2. Durante los últimos } 6 \text { meses, cuando tuvo } \\
\text { erecciones con la estimulación sexual, } \\
\text { ¿con qué frecuencia fue suficiente } \\
\text { la rigidez para la penetración? }\end{array}$ & 0 & 1 & 2 & 3 & 4 & 5 \\
\hline $\begin{array}{l}\text { 3. Durante los últimos } 6 \text { meses, al intentar } \\
\text { una relación sexual, ¿con qué frecuencia } \\
\text { logró penetrar a su pareja? }\end{array}$ & 0 & 1 & 2 & 3 & 4 & 5 \\
\hline \multirow[t]{2}{*}{$\begin{array}{l}\text { 4. Durante los últimos } 6 \text { meses, durante } \\
\text { la relación sexual, ¿con qué frecuencia } \\
\text { logró mantener la erección después } \\
\text { de la penetración? }\end{array}$} & 0 & 1 & 2 & 3 & 4 & 5 \\
\hline & $\begin{array}{l}\text { Sin } \\
\text { actividad } \\
\text { sexual }\end{array}$ & $\begin{array}{l}\text { Extrema- } \\
\text { damente } \\
\text { difícil }\end{array}$ & $\begin{array}{l}\text { Muy } \\
\text { difícil }\end{array}$ & Difícil & $\begin{array}{l}\text { Algo } \\
\text { difícil }\end{array}$ & $\begin{array}{l}\text { Sin } \\
\text { dificultad }\end{array}$ \\
\hline \multirow[t]{2}{*}{$\begin{array}{l}\text { 5. Durante los últimos } 6 \text { meses, durante } \\
\text { la relación sexual, ¿cuál fue el grado } \\
\text { de dificultad para mantener la erección } \\
\text { hasta completar la relación sexual? }\end{array}$} & 0 & 1 & 2 & 3 & 4 & 5 \\
\hline & $\begin{array}{l}\text { Muy bajo } \\
\text { o nulo }\end{array}$ & Bajo & $\begin{array}{l}\text { Modera- } \\
\text { do }\end{array}$ & Alto & $\begin{array}{l}\text { Muy } \\
\text { alto }\end{array}$ & \\
\hline $\begin{array}{l}\text { 6. Durante los últimos } 6 \text { meses, } \\
\text { ¿cómo calificaría la confianza que tiene } \\
\text { en poder lograr y mantener una erección? }\end{array}$ & 1 & 2 & 3 & 4 & 5 & \\
\hline
\end{tabular}

Actividad sexual: Incluye el acto sexual, caricias, juegos anteriores al acto y la masturbación.

Estimulación sexual: Incluye situaciones como juegos amorosos con una pareja o mirar fotos eróticas, etc.

Relación sexual: Se define como la penetración de la pareja.

Según los resultados de la suma de las seis preguntas, los pacientes quedan clasificados en:

25: no disfunción eréctil

17-25: disfunción eréctil leve

11-16: disfunción eréctil moderada

$<10$ : disfunción eréctil grave

toxicomanías, deformidad anatómica del pene, diagnóstico primario de otra disfunción sexual, priapismo, alteraciones psiquiátricas graves o retinitis pigmentaria.

Tras recibir información sobre la enfermedad y el fármaco en la consulta de demanda, a los pacientes se les pedía consentimiento informado para formar parte del estudio. Los que aceptaron participar fueron citados a una primera visita en la que se realizó una anamnesis y una exploración física que incluía toma de tensión arterial, palpación de pulsos periféricos, valoración del desarrollo mamario, de genitales y de caracteres sexuales secundarios, tacto rectal y exploración del reflejo cremastérico. Cuando no se disponía de analítica reciente (tres meses previos), se realizó hemograma y bioquími- 
ca general, incluyéndose determinaciones hormonales (prolactina, testosterona, TSH, FSH, LH y estradiol) si tras la exploración física existía sospecha de patología endocrinológica. Se recogieron variables sociodemográficas (edad, estado civil, nivel de estudios y de renta familiar), el consumo de tabaco $\mathrm{y}$ alcohol, la presencia de enfermedades concomitantes y la toma de fármacos. Además en los casos en los que la pareja accedió a participar en el estudio se midió su grado de satisfacción sexual previo a la toma del sildenafilo por el varón, mediante la pregunta: “¿cómo valora su grado de satisfacción sexual?" con una escala de propuestas de 0 a 3 (indicando 0: no satisfactoria, 1: moderadamente satisfactoria, 2: satisfactoria, 3: muy satisfactoria). Tras informarles acerca de los posibles efectos secundarios, se les instruyó sobre la posología del fármaco $(50 \mathrm{mg} /$ día, una hora antes de la actividad sexual, salvo en pacientes con insuficiencia hepática y/o renal o mayores de 65 años, que comenzaron con $25 \mathrm{mg} /$ día, con una dosis máxima de un comprimido/día) y se les prescribió para su adquisición.

En una segunda visita concertada, tras el consumo de 4 comprimidos, se readministró el cuestionario, complementado con una pregunta de eficacia global (¿mejoraron sus erecciones con el sildenafilo?) y se repitió la pregunta a la pareja después de la toma del fármaco por el varón. Asimismo se interrogó sobre la aparición de efectos secundarios mediante una pregunta abierta. Los pacientes sin mejoría subjetiva en la pregunta de eficacia global fueron citados de nuevo para evaluarlos tras tomar otros 4 comprimidos con doble dosis.

Para el análisis estadístico se utilizó el paquete SPSS, versión 9.0. Las variables descriptivas cuantitativas fueron descritas con la media y la desviación estándar y la mejoría de las puntuaciones del campo función eréctil del IIEF se midieron con el test de Wilcoxon para pruebas no paramétricas.

\section{RESULTADOS}

Se incluyeron inicialmente en el estudio 45 varones, con una edad media de 56,8 años (IC del 95\%: 32,6-81), cuyas variables sociodemográficas más destacadas se recogen en la tabla I. El grado de DE al inicio del estudio fue leve en 11 casos, moderado en 21 y grave en 13 .

En la tabla II se recogen las patologías asociadas y el consumo de fármacos y tóxicos. Las patologías más frecuentemente encontradas en nuestra muestra han sido la HTA, la dislipemia y la obesidad.

No se detectó ninguna alteración analítica que representara motivo de exclusión.

Doce sujetos (26\%) rechazaron continuar con el estudio. Los motivos por los que no iniciaron la toma del sildenafilo fueron en 5 casos por miedo a

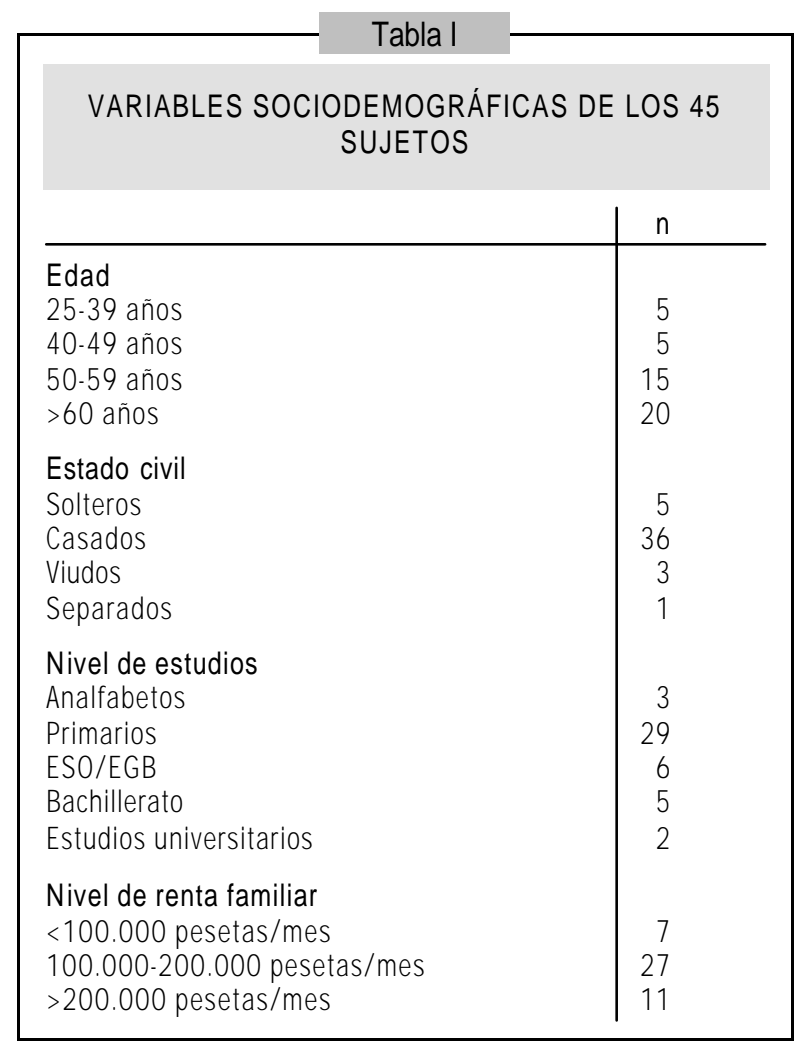

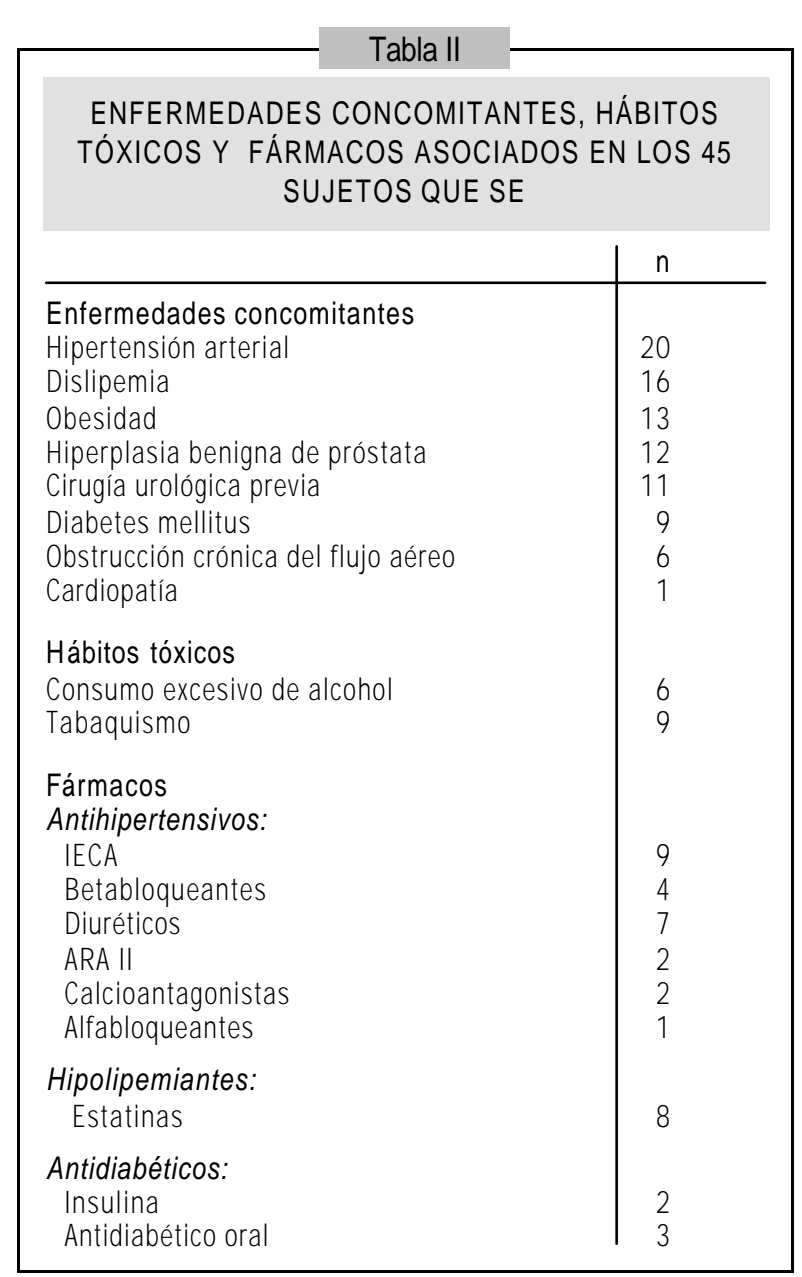


secundarismos, en 2 por mejoría espontánea tras la primera entrevista y en 5 no se pudo determinar la causa. El grado de DE en este grupo, al inicio del estudio, fue leve en 3 casos, moderada en 5 y grave en 4.

Los 33 pacientes que iniciaron el tratamiento con sildenafilo completaron el estudio. El IIEF inicial presentó como valor medio 12,73 puntos (IC 95\%: 11,22-14,24) y el IIEF final 24 (IC 95\%: 21,47-26,53), siendo esta mejoría estadísticamente significativa $(\mathrm{p}<0,0001)$. Veinte pacientes $(61 \%)$ dejaron de tener DE, según el IIEF (puntuación > $25)$. Las variaciones de las puntuaciones medias obtenidas antes y después de la toma del fármaco fueron de 18,8 a 26 puntos en las DE leves, de 13 a 26 en las DE moderadas y de 7,89 a 18,89 en las DE graves, siendo estas diferencias estadísticamente significativas. Estos datos se exponen de forma gráfica en la figura 1.

Con respecto a la pregunta de eficacia global, de los 33 pacientes que concluyeron el estudio, 28 $(84 \%)$ experimentaron mejoría subjetiva. Los 5 que no refirieron mejoría eran mayores de 50 años y con DE grave.

La dosis inicial del sildenafilo fue de $25 \mathrm{mg}$ en 4 pacientes, todos ellos $>65$ años (dos precisaron aumentar la dosis). Los 29 restantes comenzaron con $50 \mathrm{mg}$ (de los que 3 precisaron aumentar la dosis a $100 \mathrm{mg})$.

De los 15 pacientes hipertensos y de los 4 diabéticos que tomaron el fármaco, $12(80 \%)$ y $3(75 \%)$ respectivamente, respondieron al mismo.

Cinco pacientes (15\%) de los 33 que completaron el seguimiento, refirieron algún efecto secundario. Tres (9\%) comentaron cefalea y dos $(6 \%)$ rubefacción facial. En ninguno de ellos, estos efec-

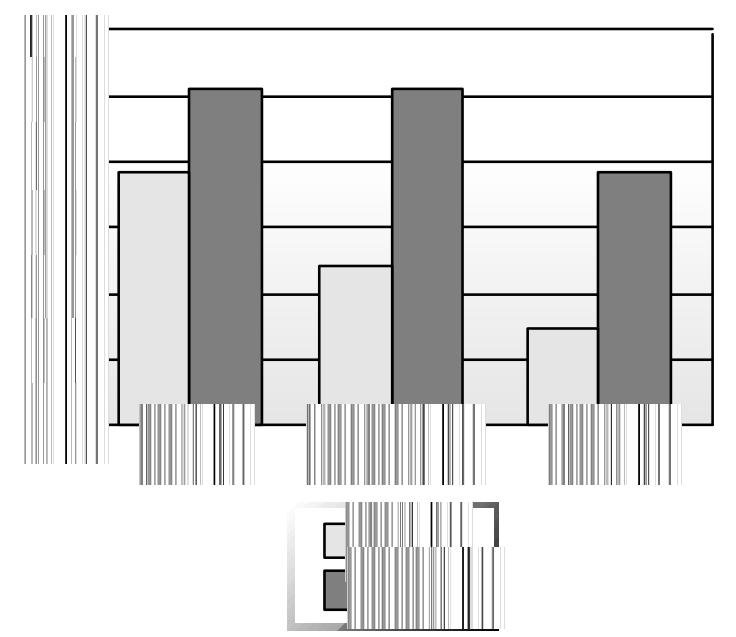

Figura 1 Comparación de las puntuaciones medias del campo función eréctil del IIEF obtenidas antes y después del tratamiento con sildenafilo $(n=33)$. tos impidieron continuar el tratamiento y cedieron espontáneamente en pocas horas.

De las 23 parejas (mujeres) que participaron, $20(87 \%)$ refirieron aumento subjetivo del grado de satisfacción sexual después de la toma del fármaco por el varón. Los datos obtenidos en las puntuaciones antes y después de la intervención, se exponen en la figura 2.

En el grupo de los doce varones que rechazaron continuar con el estudio después de realizar la primera entrevista, solamente una mujer colaboró en la encuesta.

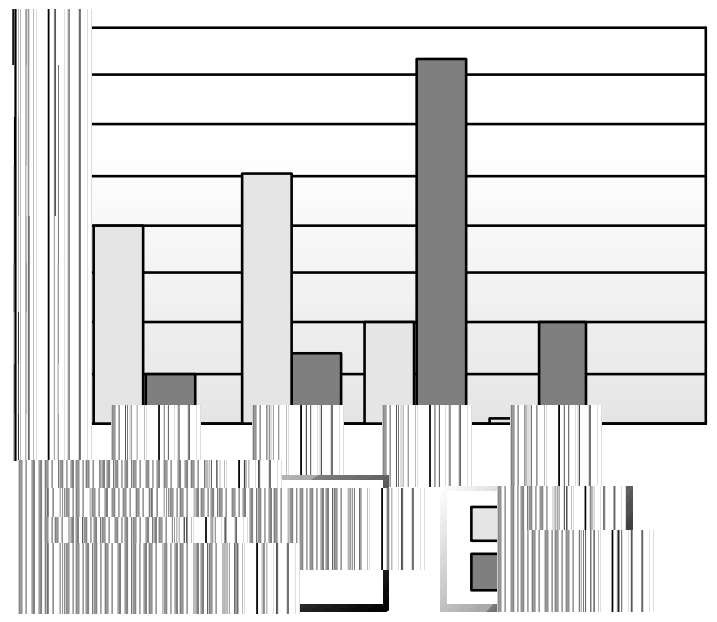

Figura 2

Comparación de las puntuaciones medias antes y después del tratamiento con sildenafilo sobre el grado de satisfacción sexual de las parejas $(n=23)$.

\section{DISCUSIÓN}

La mejoría de la DE en el $61 \%$ de los sujetos que recibieron tratamiento con sildenafilo oral es similar a la encontrada, tanto en estudios de eficacia $^{9,10}$ como de efectividad realizados en atención especializada en nuestro país ${ }^{14}$. No obstante existen determinadas características del trabajo que podrían limitar la validez de los datos obtenidos. El tamaño muestral es menor que el de otros estudios realizados a nivel especializado en Unidades de Andrología ${ }^{11,14}$. Hay que tener en cuenta que se trata de un medicamento no financiado por el Sistema Nacional de Salud, lo que unido al hecho de que, según algunos estudios, poco más del $20 \%$ de los varones discuten sus problemas sexuales con su médico ${ }^{16} \mathrm{y}$ de tratarse de una población de nivel socioeconómico medio-bajo, ha dado lugar a que pacientes que demandaban información, no aceptaran participar en el estudio, lo que dificultó el conseguir una mayor muestra. 
En los pacientes con mayor grado de DE se produjeron incrementos superiores en las puntuaciones del cuestionario, comparándolos con los casos de DE leve o moderada. Sin embargo no lograron revertir el problema manteniendo una puntuación inferior a 25 en el campo función eréctil a diferencia de lo conseguido en las DE más leves. Estos resultados han sido similares a los ensayos clínicos realizados hasta la fecha ${ }^{9,11}$.

El $80 \%$ de las parejas presentaban mejoría en el grado de satisfacción sexual; este dato parece elevado y podría estar influido tanto por una posible mala interpretación de la pregunta (en el sentido de que pensaran que la misma se refería al varón) como por la evidente subjetividad del término "relación sexual satisfactoria". El único estudio que ha evaluado el grado de satisfacción sexual de las parejas obtuvo resultados similares aunque este estudio se realizó en pacientes prostatectomizados con preservación bilateral del paquete neurovascular ${ }^{17}$.

La incidencia de efectos adversos fue similar a las de otras series ${ }^{11,18}$. No hubo casos de priapismo. Los episodios referidos fueron de intensidad leve o moderada y todos fueron dosis dependientes, cedieron en pocas horas y no impidieron continuar con el tratamiento; todos estos datos coincidentes con otros trabajos $9,11,14,18,19$.

El porcentaje de pacientes que decidieron no tomar el fármaco tras realizar la primera entrevista fue similar al estudio de Briceño y colaboradores realizado en atención especializada en nuestro país ${ }^{14}$. Un hecho a destacar en el presente estudio es que en el grupo que inició el tratamiento no se pro dujo ningún abandono, a diferencia de los ensayos clínicos en los que las pérdidas oscilaron entre el $10-13 \%{ }^{9,11,18}$. Esto podría ser explicado, al menos en parte, porque el seguimiento de estos pacientes ha sido realizado por su propio médico de familia.

El miedo a secundarismos, en parte debido a las opiniones vertidas por los medios de comunicación previas a su comercialización ${ }^{14}$ puede ser un factor limitante para su uso. Para mitigarlo se debe dar una explicación abierta, clara y detallada sobre la patología y el fármaco. También sería deseable contar siempre que se pueda con la colaboración de la pareja, dato corroborado por el hecho de que, en los doce varones que rechazaron proseguir con el estudio sólo una mujer contestó el cuestionario sobre el grado de satisfacción sexual.

Concluímos que el sildenafilo se ha mostrado como un fármaco efectivo, seguro y con pocos efectos secundarios, ofreciendo la posibilidad de un manejo sencillo para la DE en Atención Primaria. Sería deseable realizar otros estudios en este medio con un mayor número de pacientes o centrados especialmente sobre las parejas, que ayudarían a esclarecer entre otros puntos el grado de satisfacción de las mismas.

\section{AGRADECIMIENTOS}

A la Dra. Elena Andradas, epidemióloga de la Comunidad Autónoma de Madrid de la $9^{a}$ Area Sanitaria de Madrid del I NSALUD, por su valiosa contribución en el análisis estadístico de los datos y a los Dres. Juan Mejías y Miguel Téllez, urólogos del Hospital Severo Ochoa de Leganés, Madrid, por las sugerencias realizadas en el estudio.

\author{
CORRESPONDENCIA: \\ E. Cerrada Cerrada \\ C/ Barcelona $30,5^{\circ} \mathrm{B}$ \\ 28945 Fuenlabrada. Madrid \\ e-mail: ecerrada@terra.es
}

\section{Bibliografía}

1. NIH Consensus Development Panel on Impotence. JAMA 1993; 270(1): 83-90.

2. Arrondo JL. Actualización en Andrología. Pamplona: Servicio Navarro de Salud, 1999.

3. Benet $A E$, Melman A. The epidemiology of erectile dysfunction. Urol Clin North Am 1995; 22: 699-709.

4. Beutel M. Psychosomatic aspects in the diagnosis and treatment of erectile dysfunction. Andrología 1999; 31(1): 37-44.

5. Ernst E, Pittler MH. Yohimbine for erectile dysfunction: a systematic review and meta-analysis of randomized clinical trials. J Urol 1998; 159: 433-6.

6. Ramada FJ, Navalón P, Claramonte FJ, Beltrán JF, Ramos M, Zaragozá J. Impacto de la creación de las
Unidades de Andrología en el tratamiento por inyecciones intracavernosas de la disfunción eréctil. Arch Esp Urol 1999; 52 (3): 257-61.

7. Montague DK, Barada JH, Belker AM, Levine LA, Nadig PW, Roehrborn CG, et al. Clinical guidelines panel on erectile dysfunction: summary report on the treatment of organic erectile dysfunction. J Urol 1996; 156: 2007-11.

8. Padma-Nathan H, Hellstrom WJ, Kaiser FE, Labasky RF, Lue TF, Nolten WE, et al. Treatment of men with erectile dysfunction with transurethral alprostadil. $\mathrm{N}$ Engl J Med 1997; 336: 1-7.

9. Goldstein I, Lue TF, Padma-Nathan H, Rosen RC, Steers WD, Wicker PA. Oral sildenafil in the treatment 
of erectile dysfunction. N Engl J Med 1998; 338: 1397 404.

10. Padma-Nathan H, Steers WD, Wicker PA. Efficacy and safety of oral sildenafil in the treatment of erectile dysfunction: a double-blind, placebo-controlled study of 329 patients. Int J Clin Pract 52 (6): 375-9.

11. Morales A, Gingell C, Collins M, Wicker PA, Osterloch IH. Clinical safety of oral sildenafil citrate (VIAGRA) in the treatment of erectile dysfunction. Int $\mathrm{J}$ Impot Res 1998; 10: 69-73

12. Boolell M, Gepi-Attee S, Gingell JC, Allen MJ. Sildenafil, a novel effective oral therapy for male erectile dysfunction. Br J Urol 1996; 78: 257-61.

13. Girona MR, Gelado MJ, Tresserras E, Viñas A. Sildenafilo y disfunción eréctil en atención primaria. FMC 1999 6:199-205.

14. Briceño O, Egozcue S, Puigvert A, Pomerol JM. Our clinical experience with the use of sildenafil citrate to treat erectile dysfunction. Actas Urol Esp 1999; 23: 859-63.

15. Rosen RC, Cappelleri JC, Smith MD, Lipssky J, Peña
BM. Constructing and evaluating the "sexual health inventory for men: IIEF-5" as a diagnostic tool for erectile dysfunction (ED). Int J Impot Res 1998; 10(suppl 3): S35.

16. Laumann EO, Gagnon JH, Michael RT, Michaels S. The social organization of sexuality: sexuality practices in the United States. Chicago: University of Chicago Press, 1994

17. Zippe CD, Kedia AW, Kedia K, Nelson R, Agarwal A. Treatment of erectile dysfunction after radical prostatectomy with sildenafil citrate (Viagra). Urology 1998; 52: 963-6.

18. Dinsmore WW, Hodges M, Hargreaves C, Osterloh IH, Smith MD, Rosen RC. Sildenafil citrate (Viagra) in the erectile dysfunction: Near normalization in men with broad'spectrum erectile dysfunction compared with age-matched healthy control subjects. Urology 1999; 53: 800-5.

19. Marks LS, Duda C, Dorey FJ, Macairan ML, Santos PB. Treatment of erectile dysfunction with sildenafil. Urology 1999; 53(1): 19-24. 